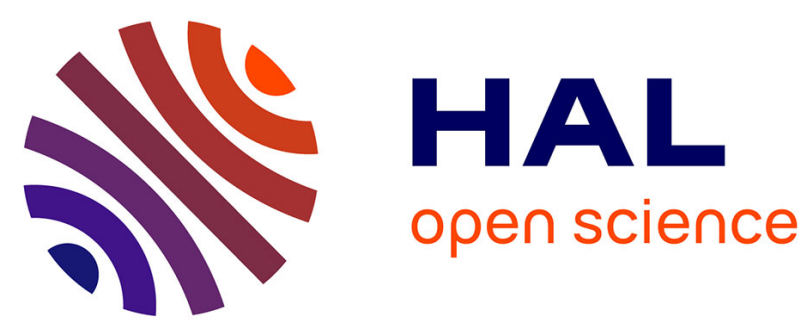

\title{
Multireflection grazing incidence diffraction used for stress measurementsin surface layers
}

Marianna Marciszko, Andrzej Baczmanski, Miroslaw Wrobel, Wilfried Seiler, Chedly Braham, Jörn Donges, Maciej Iek Sniechowski, Krzysztof

Wierzbanowskia

\section{To cite this version:}

Marianna Marciszko, Andrzej Baczmanski, Miroslaw Wrobel, Wilfried Seiler, Chedly Braham, et al.. Multireflection grazing incidence diffraction used for stress measurementsin surface layers. Thin Solid Films, 2013, 530, pp.81-84. 10.1016/j.tsf.2012.05.042 . hal-02350745

\section{HAL Id: hal-02350745 \\ https://hal.science/hal-02350745}

Submitted on 6 Nov 2019

HAL is a multi-disciplinary open access archive for the deposit and dissemination of scientific research documents, whether they are published or not. The documents may come from teaching and research institutions in France or abroad, or from public or private research centers.
L'archive ouverte pluridisciplinaire HAL, est destinée au dépôt et à la diffusion de documents scientifiques de niveau recherche, publiés ou non, émanant des établissements d'enseignement et de recherche français ou étrangers, des laboratoires publics ou privés. 


\title{
Multireflection grazing incidence diffraction used for stress measurements in surface layers
}

\author{
M. Marciszko a,*, A. Baczmański a , M. Wróbel a, W. Seiler ${ }^{\text {b }}$, C. Braham ${ }^{\text {b }}$, J. Donges ${ }^{\text {c }}$, \\ M. Śniechowski ${ }^{\text {a }}$ K. Wierzbanowski ${ }^{\text {a }}$ \\ a AGH University of Science and Technology, WFilS and WIMiIP, Al. Mickiewicza 30, 30-059 Krakow, Poland \\ ${ }^{\mathrm{b}}$ Arts et Métiers ParisTech (ENSAM), PIMM, 151, Bd de l'Hôpital 75013, Paris, France \\ ${ }^{c}$ DESY, HASYLAB, Notkestrasse 85, D-22607 Hamburg, Germany
}

Keywords:

Residual stress

X-ray diffraction

Synchrotron radiation

Grazing incidence method

\begin{abstract}
A B S T R A C T
The geometry based on the multireflection grazing incidence X-ray diffraction can be applied to measure residual stresses. Using this method, it is possible to perform a non-destructive analysis of the heterogeneous stresses for different and well defined volumes below the surface of the sample (range of several $\mu \mathrm{m}$ ). As the result, the average values of stresses weighted by absorption of X-ray radiation are measured. In this work the stress profiles as a function of penetration depth were determined for mechanically polished $\mathrm{Al}$ sample. Measurements and verification of the method were performed using classical X-ray diffractometer and synchrotron radiation with different wavelengths.
\end{abstract}

\section{Introduction}

The geometry based on the multireflection grazing incidence X-ray diffraction (named the MGIXD method) was applied to measure the gradient of residual stresses in surface layers [1-3]. When the non-symmetrical diffraction, with small incident angle $(\alpha)$, is used the penetration depth of $\mathrm{X}$ ray radiation (limited by absorption) depends mostly on the long path of incident beam (Fig. 1). However, to determine stresses it is necessary to measure interplanar spacing for different orientations of the scattering vector (characterized by $\psi$ angle). In the case of MGIXD method, the incident angle $(\alpha)$ has to be constant, therefore the diffraction angle $2 \theta$ has to be varied in order to collect data for different orientations of the scattering vector. It means, that for the same wavelength we have to measure diffraction peaks for many reflections $h k l$.

The MGIXD method was generalized and applied to measure in-depth profile of stresses $[4,5]$. In this aim the equivalent lattice parameters $<a(\phi, \psi)>_{\{h k l\}}$ are calculated from measured interplanar spacings $\langle d(\phi, \psi)\rangle_{h k l}$. Then the $\langle a(\phi, \psi)\rangle_{h k l}$ are expressed by the macrostresses $\bar{\sigma}_{i j}$ and strain free $a_{0}$ lattice constant $[4,6,7]$ :

$<a(\phi, \psi)>_{\{h k l\}}=\left[F_{i j}(h k l, \phi, \psi) \bar{\sigma}_{i j}\right] a_{0}+a_{0}$,

\footnotetext{
* Corresponding author.

E-mail addresses: marciszko@novell.ftj.agh.edu.pl (M. Marciszko), baczman@ftj.agh.edu.pl (A. Baczmański), mwrobel@agh.edu.pl (M. Wróbel), wilfrid.seiler@ensam.eu (W. Seiler), chedly.braham@paris.ensam.fr (C. Braham), joern.donges@desy.de (J. Donges), sniechowski@novell.ftj.agh.edu.pl (M. Śniechowski), wierzbanowski@fis.agh.edu.pl (K. Wierzbanowski).
}

where $F_{i j}(h k l, \phi, \psi)$ are the X-ray stress factors [2], $<a(\phi, \psi)>_{\{h k l\}}=$ $\sqrt{h^{2}+k^{2}+l^{2}}<d(\phi, \psi)>_{\{h k l\}}$ for cubic crystals, $\psi$ is the angle between scattering vector and normal to the surface, and $\phi$ is the angle between measuring direction and direction along which the principal component $\bar{\sigma}_{11}$ is defined.

In order to determine stresses, the $\bar{\sigma}_{i j}$ components of stress tensor and strain free $a_{0}$ lattice constant are treated as adjusting parameters in the least square procedure in which theoretical $\langle a(\phi, \psi)\rangle_{\{h k l\}}$ values are fitted to the experimental ones [4,6,7].

The grazing incidence X-ray diffraction is a non-destructive method which allows us to perform an analysis of the heterogeneous stress field for different volumes below the surface of the sample. In case of $\alpha \gg \alpha_{c r}$, the effective penetration depth is expressed by the formula:

$\tau=\left(\frac{\mu}{\sin \alpha}+\frac{\mu}{\sin \left(2 \theta_{\{h k l\}}-\alpha\right)}\right)^{-1}$

where $\alpha$ and $\theta$ angles are defined in Fig. 1, while $\alpha_{c r}$ is the critical angle for total external reflection $[3,8]$.

It was verified that the above approximation is fulfilled for all results presented in this work.

We obtain from measurements the mean stresses $\bar{\sigma}_{i j}(\tau)$, weighted with absorption factor:

$\bar{\sigma}_{i j}(\tau)=\int_{0}^{\infty} e^{-z / \tau} \sigma_{i j}(z) d z / \int_{0}^{\infty} e^{-z / \tau} d z$ 


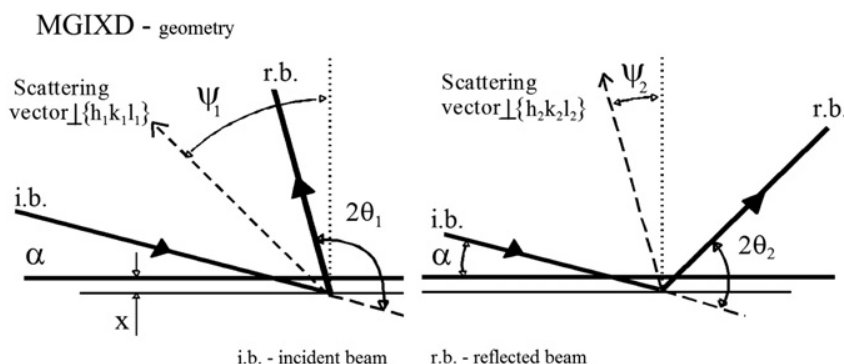

Fig. 1. Geometry of MGID method. The incidence angle $\alpha$ is fixed during measurement, while the orientation of the scattering vector is characterized by the angle $\psi$ (where $\psi\{h k l\}=\theta\{h k l\}-\alpha)$.

where $\tau$ is the penetration depth given by Eq. (2), while $z$ is the real depth under the surface.

By using a long incident beam path for small $\alpha$ angle, the above equation can be simplified, i.e.: $\tau=(\sin \alpha) / \mu$, where $\tau$ does not depend on the $\theta$ (or $\psi$ angle) and consequently the value of $\tau$ characterizing penetration depth is almost constant during stress measurement for a given incidence angle $\alpha$. The dependence of effective penetration depth calculated from Eq. (2) for MGIXD geometry is shown in Fig. 2a. It can be seen that almost constant value of $\tau$ was found for a wide range of $\psi$ angle varied during stress measurements. Moreover, the penetration depth can be changed by appropriate selection of $\alpha$ angle in order to investigate materials in different depths below the sample
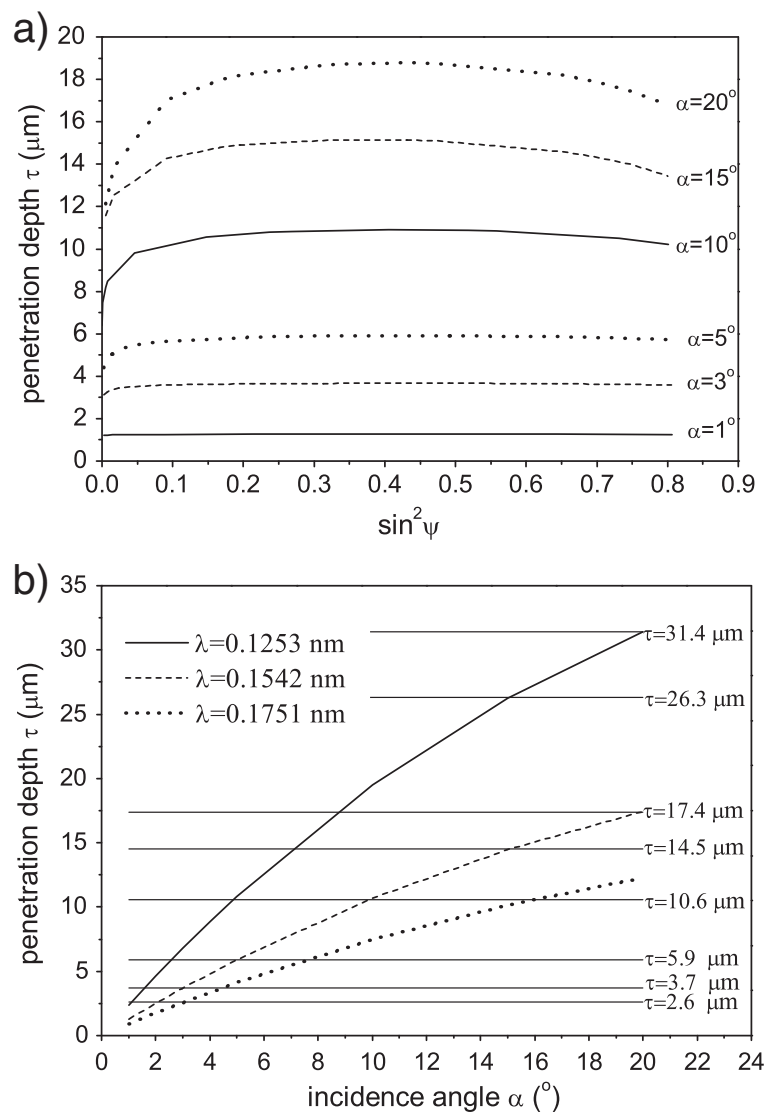

Fig. 2. The penetration depth ( $\tau$ ) vs. $\sin ^{2} \psi$ of X-rays in Al sample for different incidence angles and constant wavelength $\lambda=1.54056$ (a), and the dependence of $\tau$ vs. $\alpha$ for three different wavelengths used in the experiment (b). Calculations were performed for MGIXD geometry according to Eq. (2) $\left(\alpha \gg \alpha_{\mathrm{cr}}=0.23^{\circ}\right)$. surface (order of $\mu \mathrm{m}$ or even less than $1 \mu \mathrm{m}$ ). This gives a possibility to measure a stress gradient under the sample surface. The range of the penetration depth depends on the absorption of the used X-ray radiation in the studied material and on the incidence angle $\alpha$, usually varied between $3^{\circ}$ and $20^{\circ}$ (Fig. 2a). For $\alpha$ angle below the lower limit the measurements are difficult because of surface unevenness and also significant experimental errors which cannot be easily eliminated (for example significant peak shift due to refraction effect), while above the upper limit the available range of $\psi=\theta-\alpha$ angle is too small to determine stress from the measured equivalent lattice parameters $\left\langle a(\phi, \psi)>_{\{h k l\}}\right.$. Finally, the range of studied penetration depth can be selected choosing appropriate wavelength (energy) of the X-ray radiation, which determines the absorption in the studied material.

Although the MGIXD method is used from many years [5-7] it still needs verification and analysis of uncertainty reasons. This is the aim of this work.

\section{Measurements and analysis}

The advantages of synchrotron radiation are certainly its perfect collimation, monochromatization, high intensity and possibility of wavelength variation. Recently, synchrotron radiation (G3 beamline, DESY, Hamburg) was applied to measure the in-depth variation using the developed single reflection GIXD methods [9]. In the present work we have applied the MGIXD (multiple reflections) method on the same beamline. The aims of this work are verification of the MGIXD method by measurements with different wavelengths (synchrotron radiation) and comparison between results obtained using synchrotron radiation and standard laboratory diffractometer. For a given wavelength the penetration depth changes with the incidence angle $(\alpha)$, as shown in Fig. 1. However, using three different wavelengths we can find three corresponding incidence angles $(\alpha)$ for which the penetration depth is the same (see horizontal lines in Fig. 2b). The important question, verifying our methodology, is whether the same stresses are determined for such combination of wavelengths and incident angles.

In our experiment the Al2017 sample (aluminum alloy: $4.3 \% \mathrm{Cu}$, $0.4 \% \mathrm{Si}, 0.7 \% \mathrm{Fe}, 0.6 \% \mathrm{Mn}, 0.6 \% \mathrm{Mg}, 0.25 \% \mathrm{Zn}, 0.1 \% \mathrm{Cr}, 0.15 \% \mathrm{Ti}$, Al balance) having a surface of $1 \mathrm{~cm} \times 3.6 \mathrm{~cm}$ was studied. The material was recrystallized (initial mean grain size $120 \mu \mathrm{m}$ ) and next the surface was mechanically polished (roughness $R_{a}=0.35 \mu \mathrm{m}$ ) in order to introduce stress gradient. This specimen exhibits low crystal anisotropy (Zener factor $A=1.2$ ) and random crystallographic texture (checked using $\mathrm{X}$-ray diffraction).

At first, the MGIXD method was applied on the X-Pert Philips X-ray laboratory diffractometer $\left(\mathrm{Cu} \mathrm{K} \mathrm{K}_{\alpha}\right.$ radiation, wavelength $\lambda=$ $1.54056 \AA$ ) equipped with a Göbel mirror (divergence: $0.02^{\circ}$ in the incident beam optics), parallel-plate collimator (divergence: $0.27^{\circ}$ ) and flat graphite monochromator placed in front of proportional punctual detector. Next, the measurements were performed using a synchrotron radiation at G3 beamline at the DORIS III storage ring. In this case the parallel-plate collimator and scintillation detector were also used in the secondary beam optics, while the incident beam was monocromatized by Ge double-crystal and the higher harmonics were suppressed by gold mirror [10]. Both experimental setups ensure a parallel beam configuration minimizing effect of peak position shift due to the sample displacement and defocusing. This configuration is usually applied in the grazing incidence method. In the analysis of experimental data the diffraction peaks were corrected for the absorption, Lorentz-polarization [11,12] and reflection effects [8]. It was found that the refraction correction was important only for the incident angles $\alpha$ lower than $5^{\circ}$, while other corrections did not change significantly the values of the determined stresses.

The experimental setup was tested on the $\mathrm{Al}$ and $\mathrm{LaB}_{6}$ stress-free powder samples. The reproducibility of the MGIXD method was checked repeating measurements for different powder specimens. Example results for two Al-powders measured on X-Pert Philips 
diffractometer will be shown together with the results for the polished sample in Fig. 4. It was found that maximum value of determined fictitious stress in Al-powders is between 0 and - $15 \mathrm{MPa}$. This value can be considered as the uncertainty of measurements arising from misalignments of the diffractometer and sample position. From our experiences with powder samples and also from theoretical analysis we can also conclude that the most important source of systematic error is caused by the shift of $2 \theta$ zero value. Misalignment of the diffractometer equal $\Delta 2 \theta=0.01^{\circ}$ leads to fictitious stress of about 10-15 MPa determined for Al stress-free powder. However, this error can be minimized by careful alignment of the diffractometer or the results obtained for the studied sample can be corrected using powder diffraction data.

The $<a(\phi, \psi)>_{\{h k l\}}$ parameters were determined in function of $\sin ^{2} \psi$ plots using different reflections $h k l$ for the polished Al2017 sample (Fig. 3). In the case of synchrotron radiation the combination of different wavelengths $\left(\lambda_{1}=0.12527 \mathrm{~nm}, \lambda_{2}=0.15419 \mathrm{~nm}\right.$ and $\left.\lambda_{3}=0.17512 \mathrm{~nm}\right)$ and appropriate incidence angles $\alpha$ allowed to perform three or two stress measurements for the same penetration depth (conditions are given by intersections of the horizontal lines with $\tau$ vs. $\alpha$ plots in Fig. 2b). Using our fitting procedure (based on Eq. (1)) the $\bar{\sigma}_{11}$ stress was determined for different penetration depths $\tau$ (Fig. 4). The statistical uncertainties of the measured stresses obtained from the least square method (Eq. (1)) are shown using the error bar. In the analysis of experimental data, $\bar{\sigma}_{33}=0$ and $\bar{\sigma}_{22}=\bar{\sigma}_{11}$ were assumed for the components of stress tensor. The latter assumption was verified with X-Pert diffractometer by measuring $\langle a(\phi, \psi)\rangle_{\{h k l\}}$ vs. $\sin ^{2} \psi$ plots for two perpendicular directions (the measured components $\bar{\sigma}_{11}$ and $\bar{\sigma}_{22}$ where approximately equal for all studied penetration depths).
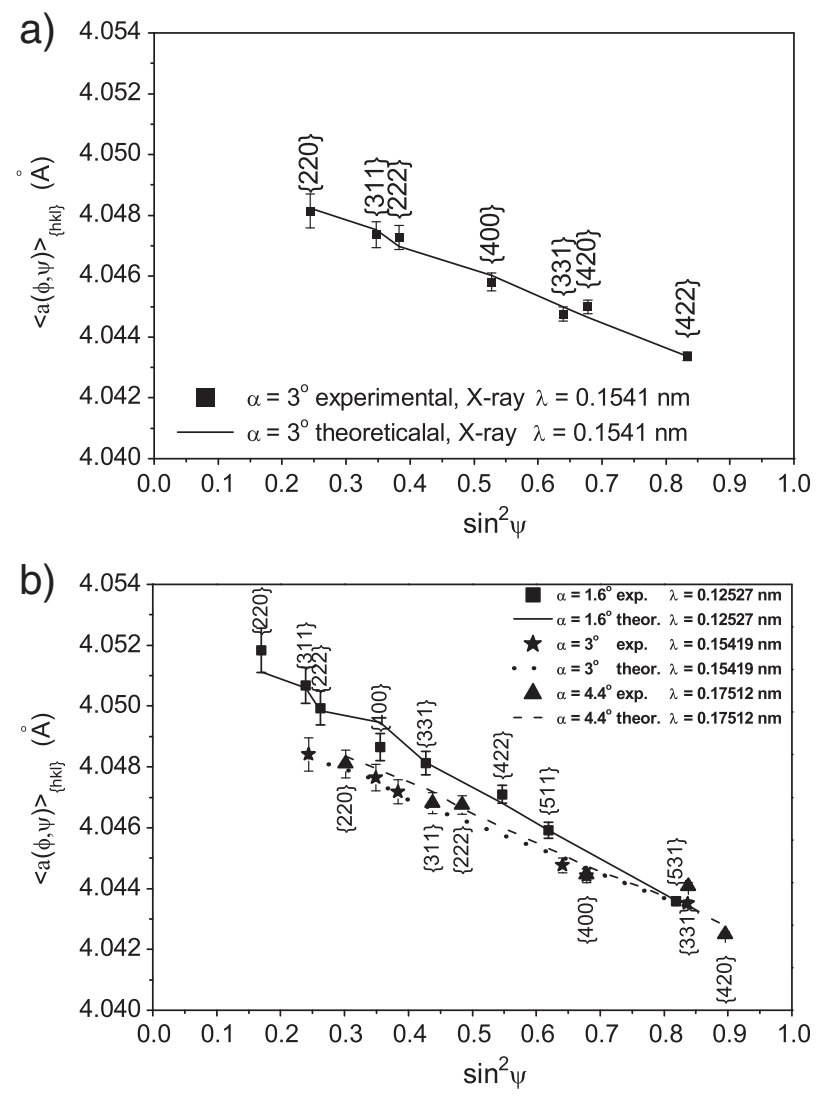

Fig. 3. Measured lattice parameters $\langle a(\phi, \psi)\rangle_{\{h k l\}}$ (points) and theoretical results of fitting (continuous lines) vs. $\sin ^{2} \psi$ for polished Al samples. Results of grazing incidence method obtained from classical diffractometer (a) and from synchrotron radiation (b) are presented. The plots are shown for the penetration depth $\tau=3.48 \mu \mathrm{m}$.

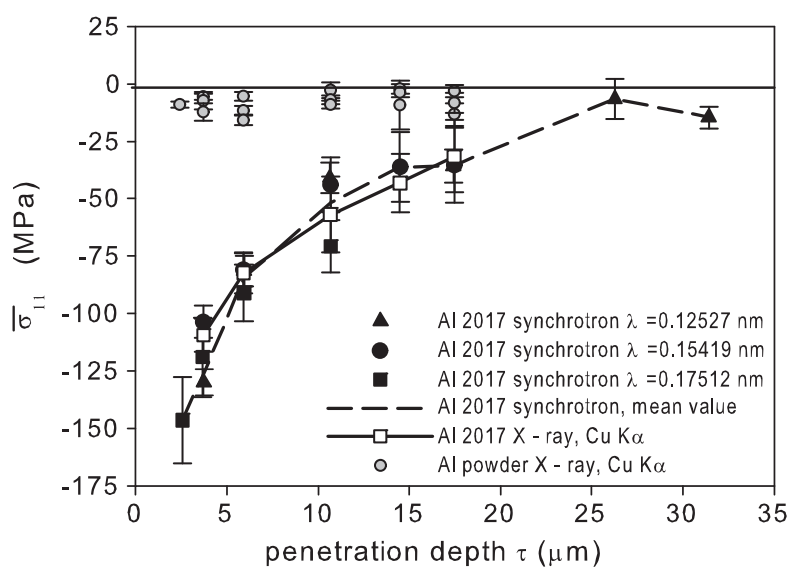

Fig. 4. Experimental $\bar{\sigma}_{11}$ stress in function of penetration depth $\tau$ for polished Al2017 sample and $\mathrm{Al}$ powder. Results obtained from laboratory diffractometer ( $\mathrm{Cu} \mathrm{K} \mathrm{K}_{\alpha}$-radiation) and using synchrotron radiation with three different wavelengths are compared.

\section{Discussion and conclusions}

The $\left\langle a(\phi, \psi)>_{\{h k l\}}\right.$ Vs. $\sin ^{2} \psi$ plots obtained for the Al2017 polished sample (Fig. 3 ) show a good agreement between results obtained using laboratory diffractometer and synchrotron radiation. In spite of different reflections $h k l$, different incident angles $\alpha$ and different wavelengths $\lambda$ used in synchrotron experiment, very similar $\langle a(\phi, \psi)\rangle_{\{h k l\}}$ vs. $\sin ^{2} \psi$ plots were obtained, when the measurements were performed for the same depth $\tau$. Therefore the correlation between measured lattice strains and penetration depth was confirmed. Moreover, the difference between $\langle a(\phi, \psi)\rangle_{\{h k l\}}$ vs. $\sin ^{2} \psi$ plots for different $\tau$ indicates strain variation in function of the depth.

Analysis of experimental data clearly shows that the differences between $<a(\phi, \psi)>_{\{h k l\}}$ vs. $\sin ^{2} \psi$ plots measured at different depths are caused by a stress gradient. In Fig. 4 the in-depth variation of determined $\bar{\sigma}_{11}$ stress is presented. As expected, a compressive plane stress decreasing with depth was introduced by plastic deformation of the sample surface during mechanical polishing. Comparison of the $\bar{\sigma}_{11}(\tau)$ profiles confirms a very good accordance between results obtained using different wavelengths of synchrotron radiation. What is more, these results perfectly agree with $\bar{\sigma}_{11}(\tau)$ profile measured using laboratory diffractometer. It should be stated that synchrotron radiation enabled to increase the range of penetration depth (2.5$32 \mu \mathrm{m})$ in comparison with classical diffractometer (3-18 $\mu \mathrm{m})$.

The main sources of uncertainties were studied using the stress free powder samples (this test must be always performed before measurements). It was found that parallel configuration of the diffractometer minimizes the sample misalignment error but special attention should be paid to the accurate alignment of the diffractometer (especially $2 \theta$ angle).

In conclusion, it should be stated that the results obtained in this work verify positively the MGIXD method. What is more, almost the same results with similar accuracy were obtained using a laboratory diffractometer equipped with a Göbel mirror and the synchrotron radiation. The advantage of the synchrotron radiation is that the range of studied depth was increased but this problem can be also partly overcome using different X-ray tubes at a classical diffractometer.

\section{Acknowledgments}

This work was financed by the Polish National Centre for Science (NCN) basing on the decision number: DEC-2011/01/B/ST8/07394 and by the Polish Ministry of Science and Higher Education (grant: 3264/B/H03/2011/40 and statutory research AGH: 11.11.110.143). 


\section{References}

1] Ch. Genzel, Phys. Status Solidi A 146 (1994) 629

[2] U. Welzel, J. Ligot, P. Lamparter, A.C. Vermeulenb, E.J. Mittemeijer, J. Appl. Crystallogr. 38 (2005) 1.

[3] A. Kumar, U. Welzel, E.J. Mittemeijer, J. Appl. Crystallogr. 39 (2006) 633.

[4] S.J. Skrzypek, A. Baczmanski, Adv. X-ray Anal. 44 (2001) 134.

[5] S.J. Skrzypek, A. Baczmanski, W. Ratuszek, E. Kusior, J. Appl. Crystallogr. 34 (2001) 427.
[6] A. Baczmański, C. Braham, W. Seiler, N. Shiraki, Surf. Coat. Technol. 182 (2004) 43.

[7] S. Wroński, K. Wierzbanowski, A. Baczmański, Powder Diffr. Suppl. 24 (2009) S11.

[8] Ch. Genzel, Mater. Sci. Technol. 21 (2005) 10.

[9] T. Erbachen, A. Wanner, T. Beck, O. Vohringer, J. Appl. Crystallogr. 41 (2007) 317.

[10] Beamline: G3 - HASYLAB - DESY, http://hasylab.desy.de/facilities/doris_iii/ beamlines/g3.

[11] B.D. Cullity, Elements of X-ray Diffraction, Addison Wesley Mass, 1978.

[12] W. Yinghua, J. Appl. Crystallogr. 20 (1987) 258. 